2009, ApJ, ??, ??

Preprint typeset using LATEX style emulateapj v. 08/22/09

\title{
THE NEWLY BORN MAGNETARS POWERING GAMMA-RAY BURST INTERNAL-PLATEAU EMISSION: ARE THERE STRANGE STARS?
}

\author{
Yun-Wei Yu ${ }^{1}$, XiaO-Feng CaO ${ }^{1}$, And Xiao-Ping Zheng ${ }^{1}$ \\ Draft version November 3, 2018
}

\begin{abstract}
The internal-plateau X-ray emission of gamma-ray bursts (GRBs) indicates that a newly born magnetar could be the central object of some GRBs. The observed luminosity and duration of the plateaus suggest that, for such a magnetar, a rapid spin with a sub- or millisecond period is sometimes able to last thousands of seconds. In this case, the conventional neutron star (NS) model for the magnetar may be challenged, since the rapid spin of nascent NSs would be remarkably decelerated within hundreds of seconds due to $r$-mode instability. In contrast, the $r$-modes can be effectively suppressed in nascent strange stars (SSs). In other words, to a certain extent, only SSs can keep nearly-constant extremely-rapid spin for a long period of time during the early ages of the stars. We thus propose that the sample of the GRB rapidly-spinning magnetars can be used to test the SS hypothesis based on the distinct spin limits of NSs and SSs.

Subject headings: gamma rays: bursts — stars: neutron
\end{abstract}

\section{INTRODUCTION}

In the standard fireball model for gamma-ray bursts (GRBs; see reviews by Piran 2005; Mészáros 2005), the GRB prompt emission is considered to arise from an internal dissipation in a relativistic expanding fireball, while the afterglow emission is produced by the deceleration of the fireball by circumburst medium, i.e., a blast wave. As understood above, it seems unlikely to directly find information about the GRB central engine, which drives the fireball, from the observed emission. However, based on some observational constraints and theoretical simulations, it is widely accepted that black holes or rapidly spinning magnetars (highly-magnetized pulsars) formed during the death of the progenitors play an important role in driving the fireball.

Furthermore, the Swift-discovered delayed intermittent bright X-ray flares demonstrate that the GRB central objects should be still very active after the bursts (Burrows et al. 2005; Yu \& Dai 2009). Analogically, the shallowdecaying segment remarkably appearing in the canonical X-ray light curve (Nousek et al 2006; Zhang et al. 2006) also strongly implies an energy injection into the GRB blast waves, which is quite likely to result from a longlasting energy release from the central objects. Under these requirements, spinning-down magnetars are usually suggested as GRB central objects in the literature (e.g., Bucciantini et al. 2009; Corsi \& Mészáros 2009; Dai 2004; Dai et al. 2006; De Pasquale et al. 2007; Zhang \& Dai 2008, 2009). The shallow-decaying afterglows can somewhat be regarded as an observational signature of rapidly spinning magntetars (Dai \& Lu 1998a, b; Yu \& Dai 2007; Zhang \& Mészáros 2001).

In particular, a nearly-constant X-ray emission followed by a very steep decline with a temporal index of $\sim 9$ was reported in GRB 070110 by Troja et al. (2007). The abrupt cutoff of the X-ray light curve robustly indicates that this plateau emission is of "internal" origin,

\footnotetext{
${ }^{1}$ Institute of Astrophysics, Huazhong Normal University, Wuhan, 430079, China; yuyw@phy.ccnu.edu.cn
}

ruling out the external shock model. Moreover, the observed luminosity and duration of the plateau are well consistent with the parameters of a newly born magnetar. So, following Zhang \& Mészáros (2001), Troja et al. (2007) further argued that this puzzling plateau could be directly produced by the internal dissipation of the magnetar-driven wind at small radii, before the energy of the wind is deposited into the GRB blast wave. Then, the observed luminosity of the plateau tracking the spindown luminosity of the GRB magnetar makes it possible to "directly" explore the properties of the central objects by using observed emission.

As a pioneering attempt, Lyons et al. (2009) investigated the magnetic and rotational properties of the central magnetars for ten GRB 070110-like GRBs. While the inferred magnetic field strengthes are basically typical for magnetars, the spin periods are usually found to be as short as the Kepler period, sometimes even at thousands of seconds after the GRB trigger. However, for a magnetar composing of normal neutron matter (i.e., neutron star; NS), it seems difficult to keep nearly-Kepler spin for more than one thousand seconds, because the spin of the nascent NS could be sorely restricted by some stellar instabilities, especially, $r$-mode instability on focus in this letter (Sá 2004; Sá \& Tomé 2005, 2006; Yu et al. 2009). In contrast, for a magnetar composing of strange quark matter (i.e., strange star; $\mathrm{SS})^{2}$, the $r$ modes-induced limit on the stellar spin is absent during the early ages of the star, because of the effective suppression of the $r$-modes by the quark matter's bulk viscosity (e.g., Wang \& Lu 1984; Madsen 1998; Zheng et al. 2006). Therefore, we argue that a newly born magnetar, which can maintain constant rapid spin for a long

\footnotetext{
2 According to the different physics of dense matter, a variety of models for compact stars have been constructed in the past forty years (see reviews by Weber 2005; Weber et al. 2006). Most of these models can be regarded as advanced versions of the conventional NS model. However, as an extreme case, SSs composing of strange quark matter (usually with a thin nuclear crust) are predicted to be completely different from ordinary NSs (e.g., Alcock et al. 1986).
} 
period of time, could be a $S S$ candidate. Based on this consideration, we propose that the sample of the GRB rapidly-spinning magnetars can be used to test the $S S$ hypothesis.

In the next section, we briefly review the magnetar model for the internal-plateau emission, and then some magnetar parameters are derived from a small GRB sample. In Sect. 3, we analysis the $r$-mode limit on the spin of NSs in the framework of a second-order $r$-mode model developed by Sá (2004). By confronting the $r$-mode limit with the GRB magnetar sample, we makes an attempt to find SS candidates. Finally, a summary and discussion are given in Sect. 4.

\section{MAGNETARS POWERING INTERNAL PLATEAUS}

As discussed above, the internal-plateau emission indicates that a rapidly spinning magnetar has been formed as a central object during the prompt phase of some GRBs. The initial spin-down of a magnetar could be very complicated during the first tens of seconds after its birth. On the longer timescales that we are interested in, some short-term processes such as neutrino-driven winds could be no longer important. We here simply consider that the nascent magnetar spins down through magnetic dipole radiation as

$$
\frac{d P}{d t}=\frac{P}{\tau_{m}},
$$

where $P$ is the spin period of the star. The magnetic braking timescale reads (Shapiro \& Teukolsky 1983)

$$
\tau_{m}=\frac{6 c^{3}}{(2 \pi)^{2}} \frac{I P^{2}}{B^{2} R^{6}}=4 \times 10^{3} I_{45} R_{6}^{-6} B_{15}^{-2} P_{-3}^{2} \mathrm{~s},
$$

where $c$ is the speed of light, $I, R$, and $B$ are the inertial of moment, the radius, and the surface magnetic field strength of the magnetar, respectively. The convention $Q_{x}=Q / 10^{x}$ is adopted in cgs units hereafter. Eq. (1) yields $P(t)=P_{i}\left(1+t / T_{m}\right)^{1 / 2}$, where $P_{i}$ is the initial spin period and

$$
T_{m}=\frac{P_{i}^{2}}{2 P^{2}} \tau_{m}=\frac{1}{2} \tau_{m, i}=2 \times 10^{3} I_{45} R_{6}^{-6} B_{15}^{-2} P_{i,-3}^{2} \mathrm{~s} .
$$

The expression of $P(t)$ shows that a nascent magnetar only against the magnetic braking effect can keep nearly-constant spin for a period of time of $T_{m}$. As the spinning down, the magnetar releases the spining energy and drives an outwards-propagating wind. We then approximately estimate the luminosity of the wind by the magnetically spin-down luminosity as $L_{w} \approx L_{m s d}=$ $(2 \pi)^{2} I \dot{P} / P^{3}=\tilde{L}\left(1+t / T_{m}\right)^{-2}$ with

$$
\tilde{L} \equiv \frac{(2 \pi)^{4}}{6 c^{3}} \frac{B^{2} R^{6}}{P_{i}^{4}}=10^{49} R_{6}^{6} B_{15}^{2} P_{i,-3}^{-4} \operatorname{erg~s}^{-1} .
$$

Since the observed internal-plateau emission is deemed to be directly emitted by magnetar winds, the model quantities $\tilde{L}$ and $T_{m}$ can be easily determined by the observational isotropically-equivalent luminosity $L_{\gamma, i \text { so }}$ and the observational duration $T_{p}^{o b s}$ of the plateaus as follows

$$
\tilde{L} \geq L_{\gamma, b}=f_{b} L_{\gamma, i s o}, \quad T_{m} \geq T_{p}=\frac{T_{p}^{o b s}}{(1+z)}
$$

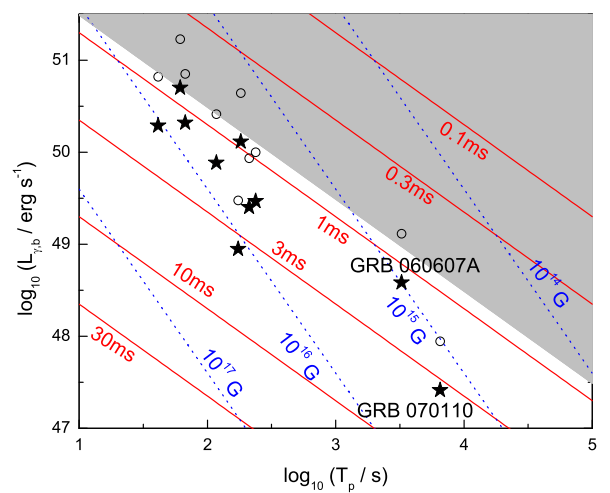

FIG. 1. - Equal- $B$ (dotted lines) and equal- $P_{i}$ (solid lines) contours determined by Eqs. (8) and (9), respectively. The observational internal-plateau data of ten GRBs are marked by the open circles for isotropic winds and solid asterisks for $\theta_{w}=45^{\circ}$. The shaded region is forbidden for magnetars due to $P_{i}<P_{K}$.

TABLE 1

THE OBSERVED PROPERTIES OF THE INTERNAL PLATEAUS OF TEN GRBs*.

\begin{tabular}{ccccc}
\hline GRB & $L_{\gamma, \text { iso }}\left(\mathrm{erg} \mathrm{s}^{-1}\right)$ & $T_{p}^{\text {obs }}(\mathrm{s})$ & $z$ & $T_{p}(\mathrm{~s})$ \\
\hline 080310 & $2.6 \mathrm{e}+50$ & 401.9 & 2.426 & 117.3 \\
071021 & $6.6 \mathrm{e}+50$ & 248.3 & 5.0 & 41.4 \\
$070721 \mathrm{~B}$ & $3.0 \mathrm{e}+49$ & 802.9 & 3.626 & 173.6 \\
070616 & $4.4 \mathrm{e}+50$ & 585.6 & 2.22 & 181.9 \\
070129 & $8.6 \mathrm{e}+49$ & 683.0 & 2.22 & 212.1 \\
070110 & $8.8 \mathrm{e}+47$ & 21887.1 & 2.352 & 6529.6 \\
$060607 \mathrm{~A}$ & $1.3 \mathrm{e}+49$ & 13294.7 & 3.082 & 3256.9 \\
$060510 \mathrm{~B}$ & $1.7 \mathrm{e}+51$ & 362.9 & 4.9 & 61.5 \\
060202 & $1.0 \mathrm{e}+50$ & 766.0 & 2.22 & 237.9 \\
050904 & $7.1 \mathrm{e}+50$ & 488.8 & 6.29 & 67.1 \\
\hline
\end{tabular}

*See Lyons et al. (2009) for a detailed explanation of the data.

where $z$ is the redshifts of the GRBs. The beaming factor $f_{b}=\left(1-\cos \theta_{w}\right)$ with $\theta_{w}$ being the half-opening angle of the winds is introduced because the magnetar winds could be collimated rather than perfectly isotropic (Bucciantini et al. 2007; Lyons et al. 2009). By solving Eq.(5), the magnetic field strengthes and the initial spin periods of the GRB magnetars can be derived as

$$
\begin{aligned}
& B \leq 2 \times 10^{15} I_{45} R_{6}^{-3} L_{\gamma, b, 49}^{-1 / 2} T_{p, 3}^{-1} \text { Gauss } \\
& P_{i} \leq 1.4 \times 10^{-3} I_{45}^{1 / 2} L_{\gamma, b, 49}^{-1 / 2} T_{p, 3}^{-1 / 2} \mathrm{~s} .
\end{aligned}
$$

The greater-than and less-than signs appear in Eqs. (5)(7), because the radiation efficiency of the winds should be lower than $100 \%$ and the cutoff of the plateaus could be caused by a rapid decrease of the stellar magnetic fields (Troja et al. 2007) or by a collapse of the magnetars into black holes (Lyons et al. 2009). In this letter, for simplicity, we only consider the case of equality for Eqs. (6) and (7) as an extreme case, as did in Lyons et al. (2009).

In order to conveniently estimate the values of $B$ and $P_{i}$ for specific GRBs in the $\lg L_{\gamma, b}-\lg T_{p}$ panel, we plot in Fig. 1 a set of dashed lines for different values of $B$ and a set of dotted lines for different values of $P_{i}$, which 
are respectively determined by

$$
\begin{aligned}
& \lg L_{\gamma, b, 49}=-2 \lg T_{p, 3}+\left(0.6-2 \lg B_{15}\right), \\
& \lg L_{\gamma, b, 49}=-\lg T_{p, 3}+\left(0.3-2 \lg P_{i,-3}\right),
\end{aligned}
$$

where a typical value of unit is adopted for $I_{45}$ and $R_{6}$ in view of the sufficiently small variations of these parameters. For a realistic magnetar, its rotation obviously cannot be rapider than the Kepler rotation, at which the star starts shedding mass at the equator. So an absolute upper limit on the spin periods of magnetars is given by the Kepler period (Haensel et al. 2009)

$$
P_{K}=C\left(\frac{M}{1.4 M_{\odot}}\right)^{-1 / 2} R_{6}^{3 / 2} \mathrm{~s}
$$

where $M$ is the gravitational mass of the magnetar. For the prefactor $C$, the careful numerical calculation given by Haensel et al. (2009) showed that its value is slightly dependent on the equation of state of the stellar matter, specifically, $C=0.783 \mathrm{~ms}$ for NSs and $0.735 \mathrm{~ms}$ for SSs. For a conservative discussion, we simply take $C=0.8 \mathrm{~ms}$ for both NSs and SSs as proposed by Lattimer \& Prakash (2004).

In Fig. 1 we also scatter the observational internalplateau data of ten GRBs as listed in Table 1, which survived from an internal-plateau test in Lyons et al. (2009) with three criterions: (1) the X-ray light curve could not be adequately fitted by the Willingale model (Willingale et al. 2007); (2) a relatively constant X-ray flux lasts for a significant period of time; and (3) the plateau is followed by a convincing steep decline with a temporal index $>4$, so that the internal origin of the plateau is favored. Under the isotropic assumption for the magnetar winds, the data (open circles) is obviously in contradiction with the Kepler limit. So as suggested by Lyons et al. (2009), we should adjust the vertical locations of the data to fit the requirement of the Kepler limit, by varying the value of the remaining free parameter $\theta_{w}$. Then we get $\theta_{w}=45^{\circ}\left(f_{b}=0.3\right)$ by assuming the fastest possible period as the Kepler period. Taking this angle as the beaming angle for each GRB, we replot the observational data using solid asterisks in Fig. 1.

\section{3. $R$-MODE LIMIT ON NSS AND IDENTIFYING SSS}

From Fig. 1, the magnetic field strengthes can be found to be around typical values of $\sim 10^{15}-10^{16}$ Gauss for magnetars. Besides that, we especially notice that a long-lasting rapid spin with a period of $\sim 1-3 \mathrm{~ms}$ is required for the magnetars in GRBs 060607A and 070110. However, for a perfectly fluid compact star with arbitrary rotation, $r$-mode oscillation can arise due to the action of the Coriolis force with positive feedback and tends to be unstable (Andersson 1998; Friedman \& Morsink 1998), succumbing to gravitational radiation-driven Chandrasekhar-Friedman-Schutz instability (Chandrasekhar 1970; Friedman \& Schutz 1978). As a result, the rapid spin of newly born NSs can be reduced early and remarkably by the strong gravitational radiation, but cannot for nascent SSs. Then, the question arises as to whether the GRB rapidly-spinning magnetars are NSs or SSs, or whether we can find SS candidates from GRB magnetars.

Following a phenomenological model for $r$-modes proposed by Sá (2004), the evolution of the amplitude of the $r$-mode oscillation, $\alpha$, can be calculated from (Owen et al. 1998; Sá \& Tomé 2005; Yu et al. 2009)

$$
\left.\frac{d \alpha}{d t}=\left[1+\frac{2 \alpha^{2}}{15}(\delta+2)\right] \frac{\alpha}{\tau_{g}}-\left[1+\frac{\alpha^{2}}{30}(4 \delta+5)\right] \frac{\alpha}{\tau_{v}}+\frac{\alpha}{2 \tau_{m}}\right)
$$

where $\delta$ is a free parameter describing the initial degree of the differential rotation of a nascent magnetar,

$$
\tau_{g}=37\left(P / P_{K}\right)^{6} \mathrm{~s}
$$

is the gravitational radiation timescale, and $\tau_{v}=\left(\tau_{s v}^{-1}+\right.$ $\left.\tau_{b v}^{-1}\right)^{-1}$ is the viscous damping timescale with $\tau_{s v}$ and $\tau_{b v}$ corresponding to the shear and bulk viscosities, respectively. Accordingly, the spin-down of the magnetar against both gravitational and magnetic braking effects is determined by

$$
\frac{d P}{d t}=\frac{4 \alpha^{2}}{15}(\delta+2) \frac{P}{\tau_{g}}-\frac{\alpha^{2}}{15}(4 \delta+5) \frac{P}{\tau_{v}}+\frac{P}{\tau_{m}} .
$$

In the case of $\tau_{g} \gg \tau_{m}$ where the $r$-modes cannot arise sufficiently rapidly, the above equation can be well approximated by Eq.(1). In contrast, for $\tau_{g} \ll \tau_{m}$, the spindown is instead dominated by the gravitational braking, if the $r$-mode amplitude can reach its saturated value in time under a condition of $\tau_{g} \ll \tau_{v}$. However, the value of $\tau_{v}$ is sensitive to the properties of the stellar matter.

To be specific, we display the viscous damping timescales for a NS and a SS respectively as (Owen et al. 1998; Madsen 2000) ${ }^{3}$ :

$$
\begin{aligned}
\tau_{s v}^{N S} & \approx 2.52 \times 10^{10} \mathcal{T}_{10}^{8} \mathrm{~s}, \\
\tau_{b v}^{N S} & \approx 1.57 \times 10^{3} \mathcal{T}_{10}^{-6}\left(P / P_{K}\right)^{2} \mathrm{~s} \\
\tau_{s v}^{S S} & \approx 2.49 \times 10^{10} \mathcal{T}_{10}^{5 / 3} \mathrm{~s}, \\
\tau_{b v}^{S S} & \approx 0.02 \mathcal{T}_{10}^{-2}\left(P / P_{K}\right)^{2} \mathrm{~s},
\end{aligned}
$$

where $\mathcal{T}$ is the stellar temperature. For a nascent magnetar with $\mathcal{T}=\mathcal{T}_{i} \sim 10^{10} \mathrm{~K}$ and $P=P_{i} \sim P_{K}$, on one hand, the shear viscous damping of the $r$-modes can be neglected undoubtedly, no matter whether the star is a $\mathrm{NS}$ or a SS due to $\tau_{s v} \gg\left(\tau_{g}, \tau_{m}, \tau_{b v}\right)$. On the other hand, the significant difference between $\tau_{b v}^{N S}\left(\gg \tau_{g}\right)$ and $\tau_{b v}^{S S}\left(\ll \tau_{g}\right)$ would lead to two distinct results: (1) For a nascent SS, the $r$-mode instability is suppressed effectively by the bulk viscosity. By equaling $\tau_{g}$ to $\tau_{b v}^{S S}$ to get $\mathcal{T}_{10} \sim 0.02$ and following the direct-Urca-processdominated cooling $\mathcal{T}=\mathcal{T}_{i}\left(1+t / \tau_{c}\right)^{-1 / 4}$ with $\tau_{c} \sim 1 \mathrm{~s}$, we can find that it should take about $\sim 10^{7} \mathrm{~s}$ to achieve $\tau_{g} \sim \tau_{b v}^{S S}$ at which $r$-modes can arise. During this long period of time of $\sim 10^{7} \mathrm{~s}$, the spin-down of the SS is exclusively dominated by the relatively stronger magnetic dipole radiation (Zheng et al. 2006). Therefore, the upper limit of the spin periods of nascent SSs can be simply

3 The viscous timescales of the SS presented here are obtained for a normal quark matter phase. More generally, some color superconducting phases such as the 2-flavor color superconductivity and color-flavor-locked phases have also been suggested for sufficiently high density and sufficiently low temperature (see a recent review by Alford et al. 2008). In these phases, the viscosities of the quark matter can be changed (generally reduced) significantly. However, at high temperatures of $\sim 10^{10}-10^{11} \mathrm{~K}$ as in nascent magnetars concerned in this letter, the bulk viscosity of the superconducting matter could be still comparable to that of the unpaired quark matter (e.g., Alford \& Schmitt 2007). 


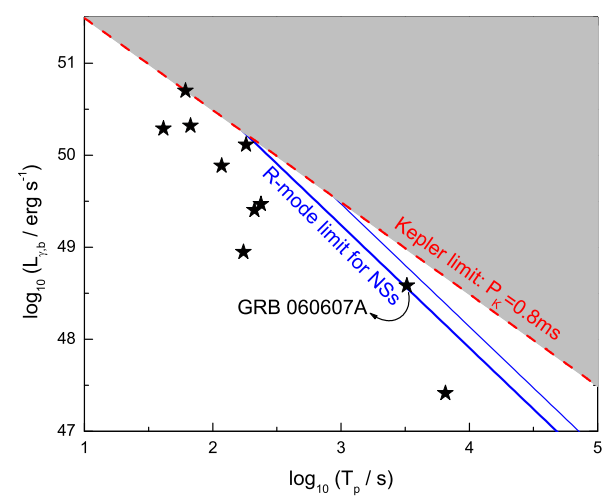

FIG. 2.- The $r$-mode limit on the spin of NSs for $T_{K}=170 \mathrm{~s}$ (thick solid line) and $T_{K}=680 \mathrm{~s}$ (thin solid line). The Kepler limit is represented by the thick dashed line, above which the shaded region is forbidden for magnetars. The beaming angle is taken to be $\theta_{w}=45^{\circ}$ for the observational data in order to make the fastest period just longer than the Kepler period.

set at the Kepler period. (2) In contrast, for nascent NSs, $r$-modes can arise rapidly and arrive at its saturated amplitude within hundreds of seconds (Sá \& Tomé 2005, 2006). As a result, the spin-down of the NSs would be first dominated by the gravitational braking, much before the magnetic braking operates (Yu et al. 2009).

Now let us investigate how long a nascent NS against $r$-mode instability can keep constant spin with $\tau_{g} \ll \tau_{m}$. In view of $\tau_{g} \ll\left(\tau_{m}, \tau_{v}^{N S}\right)$, Eqs. (11) and (13) can be solved analytically by ignoring the magnetic and viscous terms. For convenience, we here exhibit an asymptotic solution as (Sá \& Tomé 2005, 2006)

$$
P(t) \approx \begin{cases}P_{i}\left[1-\frac{2 \alpha_{i}^{2}}{15}(\delta+2) \exp \left(\frac{2 t}{\tau_{g, i}}\right)\right]^{-1} & , \text { for } t<T_{g} \\ 1.6 P_{i}\left(\frac{t}{\tau_{g, i}}\right)^{1 / 5}, & \text { for } t>T_{g}\end{cases}
$$

where $\alpha_{i}$ is the initial $r$-mode amplitude. The break time $T_{g}$ can be solved from $\left(d^{2} \alpha / d t^{2}\right)_{t=T_{g}}=0$ to be

$$
\begin{aligned}
T_{g} & =-37\left[\ln \alpha_{i}+\frac{1}{2} \ln (\delta+2)+\frac{1}{2} \ln \frac{6}{5}\right]\left(\frac{P_{i}}{P_{K}}\right)^{6} \\
& \equiv T_{K}\left(\alpha_{i}, \delta\right)\left(\frac{P_{i}}{P_{K}}\right)^{6},
\end{aligned}
$$

at which $P=1.125 P_{i}$. After $T_{g}$, the spin period of the NS can no longer be regarded as a constant and the luminosity of the wind evolves as $L_{w} \approx L_{m s d} \propto t^{-4 / 5}$, which obviously deviates from a plateau emission. So we conclude that a nascent $N S$ with an initial period $P_{i}$ can keep nearly constant spin for at most a period of time of $T_{g}\left(P_{i}\right)$. For $P_{i}=P_{K}$, we have $T_{g}=T_{K}$. Within a wide parameter region of $10^{-10}<\alpha_{i}<10^{-6}$ and $0<\delta<10^{8}$ (Sá \& Tomé 2005, 2006), the value of $T_{K}$ varies from 170 $\mathrm{s}$ to $840 \mathrm{~s}$.

Therefore, for GRB magnetars with $T_{p}>10^{3} \mathrm{~s}$, it is necessary to test whether their spin is sufficiently slow to determine a sufficiently long $T_{g}$ as

$$
P_{i}<P_{R}=P_{K}\left(T_{p} / T_{K}\right)^{1 / 6} .
$$

If not, the magnetar is quiet unlikely to be a NS. Alternatively, it could be a SS candidate. Substituting Eq.(20) into (9) with $P_{K,-3}=0.8$, we can obtain

$$
\lg L_{\gamma, b, 49}=-\frac{4}{3} \lg T_{p, 3}+\left(-0.5+\frac{1}{3} \lg T_{K}\right) .
$$

With a certain value of the free parameter $T_{K}$, this equation determines a $r$-mode limit line for NSs in the $\lg L_{\gamma, b}-\lg T_{p}$ panel, as shown by the solid lines in Fig. 2 , above which $T_{p}>T_{g}$.

Then an especial region below the Kepler limit line and above the r-mode limit line is highlighted in the $\lg L_{\gamma, b}-\lg T_{p}$ panel, where only SSs can appear. When we use this SS-only region to cover the observational GRB internal-plateau data, we can obtain that either (1) some SS candidates are indicated by the data locating in the SS-only region, or (2) all data are clearly below the $r$ mode limit line. In the latter case, the SS model cannot be in principle ruled out, but the influence of the gravitational radiation-driven $r$-mode instability on the spin of NSs would be confirmed. For the small sample of GRB magnetars listed in Table 1, we find in Fig. 2 that GRB 060607A locates just above the $r$-mode limit line, by assuming the fastest possible period as the Kepler period $\left(\theta_{w}=45^{\circ}\right)$ and taking $T_{K}=170 \mathrm{~s}$. This implies that the magnetar in GRB 060607A could be a SS. It is fair to say, though, that the uncertainties and approximations involved could be large enough that the NS model might be still available for this magnetar. So a much richer GRB sample is sorely demanded in order to get a more definite conclusion.

\section{SUMMARY AND DISCUSSION}

The internal-plateau emission is considered to indicate that a central rapidly-spinning magnetar is formed during some GRBs. Such a newly born magnetar is sometimes required to be able to keep a rapid spin with a subor millisecond period for thousands of seconds. Then, according to the spin limit of nascent NSs due to $r$-mode instability, we propose a method to identify SS candidates from the GRB magnetars, since the $r$-mode instability cannot operate in nascent SSs. Despite the smallness of the present GRB magnetar sample, the effectiveness of the method is somewhat exhibited by the appearance of GRB 060607A.

A few decades ago, the concept of SS was proposed formerly as a new and novel class of compact stars (Alcock et al. 1986; Haensel et al. 1986) and even as GRB central objects (Cheng \& Dai 1996, 1998; Dai \& Lu 1998a), based on a conjecture that the true ground state of matter is strange quark matter rather than $\mathrm{Fe}^{56}$ (Witten 1984). In both physics and astronomy, it is of significant importance to identify or rule out SSs from observational astrophysical objects. Seasonably, a plausible method was designed by Madsen (1998) according to the distinct rotational properties of NSs and SSs against the $r$-mode instability. However, for most detected Galactic millisecond pulsars, the stellar temperature is generally much lower than $\sim 10^{10} \mathrm{~K}$, in which case some complications arise from the shear viscosity due to the stellar crust (e.g., Bildsten \& Ushomirsky 2000) and the superfluidity appearing in the stellar core. This makes it difficult to distinguish SSs from NSs within the Galactic 
pulsar sample (Madsen 2000). In contrast, the situation in newly born pulsars would be much clearer due to their sufficiently high temperature. Moreover, for GRB magnetars, a relatively richer sample could be selected from the huge GRB storage, in contrast to the rare observation of newly born pulsars in the Galaxy. In view of these advantages, it is worthwhile to use the newly-born GRB magnetars to test the SS hypothesis.

However, it should be notice that the uncertainties and approximations of the model still prohibit the present small GRB magnetar sample from becoming a gold sample for testing SS hypothesis. Therefore, it is demanded to make great efforts in following aspects: (1) a thorough investigation on magnetar wind. Here we would like to point out that the angle of the magnetar wind is probably different from that of the GRB ejecta, in respect that the GRB ejecta could be driven by an hyperaccretion onto the magnetar rather than the magnetar wind (Zhang \& Dai 2008, 2009). (2) finding other constraints on the wind angle. For example, in order to build up sufficient dynamo action responsible for the intense magnetic fields, the initial spin periods of magentars could be required to be $\leq 10 \mathrm{~ms}$ (Lyons et al. 2009; Usov 1992), which leads to $\theta_{w}>16^{\circ} ;(3)$ accumulating a much larger number of GRB magnetars.

We would like to acknowledge Z.G. Dai, K.S. Cheng, and Xue-Wen Liu for their useful suggestions. This work is supported by the National Natural Science Foundation of China (grant no. 10773004).

\section{REFERENCES}

Alcock, C., Farhi, E., \& Olinto, A. 1986, ApJ, 310, 261

Alford, M.G., Rajagopal, K., Schaefer, T., Schmitt., A. 2008, Rev. Mod. Phys., 80, 1455

Alford, M.G., \& Schmitt., A. 2007, AIP Conf. Proc., 964, 256

Andersson, N. 1998, ApJ, 502, 708

Bildsten, L., \& Ushomirsky, G., 2000, ApJ, 529, L33

Bucciantini, N., Quataert, E., Arons, J., et al. 2007, MNRAS, 380,1541

Bucciantini, N., Quataert, E., Metzger, B.D., et al. 2009, MNRAS, submitted, arXiv: 0901.3801

Burrows, D. N., Romano, P. Falcone, A., et al. 2005, Science, 309, 1833

Chandrasekhar, S. 1970, Phys. Rev. Lett., 24, 611

Cheng, K. S., \& Dai, Z. G. 1996, Phys. Rev. Lett., 77, 1210

Cheng, K. S., \& Dai, Z. G. 1998, Phys. Rev. Lett., 80, 18

Corsi, A., \& Mészáros, P. 2009, ApJ, 702, 1171

Dai, Z. G., 2004, ApJ, 606, 1000

Dai, Z. G., \& Lu, T. 1998a, Phys. Rev. Lett., 81, 4301

Dai, Z. G., \& Lu, T. 1998b, A\&A, 333, L87

Dai, Z. G., Wang, X. Y., Wu, X. F., \& Zhang, B. 2006, Science, 311, 1127

De Pasquale, M., Oates, S. R., Page, M. J., et al. 2007, MNRAS, 377,1638

Friedman, J. L., \& Morsink, S. M. 1998, ApJ, 502, 714

Friedman, J. L., \& Schutz, B. F. 1978, ApJ, 221, 937

Haensel, P., Zdunik, J. L., Schaefer, R. 1986, A\&A, 160, 121

Haensel, P., Zdunik, J. L., Bejger, M., Lattimer, J.M. 2009, A\&A, 502,605

Lattimer, J.M., \& Prakash, M. 2004, Science, 304, 536

Lyons, N., O'Brien, P. T., Zhang, B., et al. 2009, MNRAS, accepted, arXiv: 0908.3798

Madsen, J. 1998, Phys. Rev. Lett., 81, 3311

Madsen, J. 2000, Phys. Rev. Lett., 85, 10

Mészáros, P. 2006, Rep. Prog. Phys., 69, 2259
Nousek, J. A., Kouveliotou, C., Grupe, D., et al. 2006, ApJ, 642, 389

Owen, B. J., Lindblom, L., Cutler, C., et al. 1998, Phys. Rev. D, 58,084020

Piran, T. 2005, Rev. Mod. Phys., 76, 1143

Rees, M. J., \& Mészáros, P. 1998, ApJ, 496, L1

Sá, P. M. 2004, Phys. Rev. D, 69, 084001

Sá, P. M. \& Tomé, B. 2005, Phys. Rev. D, 71, 044007

Sá, P. M. \& Tomé, B. 2006, Phys. Rev. D, 74, 044011

Shapiro, S. L., \& Teuklosky, S. A. 1983, Black holes, white dwarfs and neutron stars (New York, Wiley)

Troja, E., Cusumano, G., O'Brien, P. T., et al. 2007, ApJ, 665, 599

Usov, V. V. 1992, Nature, 357, 472

Wang, Q. D., \& Lu, T. 1984, Phys. Lett. B, 148, 211

Weber, F., 2005, Prog. Part. Nucl. Phys. 54, 193

Weber, F., Negreiros, R., \& Rosenfield, P. 2006, Invited review talk presented at "Neutron Stars and Pulsars: About 40 years after the discovery", Physics Center Bad Honnef, Germany, 14-19 May arXiv: 0705.2708

Willingale, R., O'brien, P. T., Osborne, J. P., et al. 2007, ApJ, 662,1093

Witten, E. 1984, Phys. Rev. D, 30, 272

Yu, Y. W., Cao, X. F., \& Zheng, X. P. 2009, Research in Astro. and Astrophys., 9, 1024

Yu, Y. W., \& Dai, Z. G. 2007, A\&A, 470, 119

Yu, Y. W., \& Dai, Z. G. 2009, ApJ, 692, 133

Zhang, B., Fan, Y. Z., Dyks, J., et al. 2006, ApJ, 642, 354

Zhang, B., \& Mészáros, P. 2001, ApJ, 552, L35

Zhang, D., \& Dai, Z. G. 2008, ApJ, 683, 329

Zhang, D., \& Dai, Z. G. 2009, ApJ, 703, 461

Zheng X. P., Yu Y. W., \& Li J. R. 2006, MNRAS, 369, 376 